\title{
Variation of Quantum Efficiency in CZTSSe Solar Cells with Temperature and Bias Dependence by SCAPS Simulation
}

\author{
Sanghyun Lee ${ }^{1}$ and Kent J. Price ${ }^{2}$ \\ 1. School of Engineering and Information Systems, Morehead State University, Morehead, KY 40351, USA \\ 2. Department of Math and Physics, Morehead State University, Morehead, KY 40351, USA
}

Received: October 26, 2016 / Accepted: November 08, 2016 / Published: February 28, 2017.

\begin{abstract}
The quantum efficiency of CZTSSe (copper zinc tin sulphur selenium) thin film solar cells is numerically simulated at different temperatures and under a set of bias conditions about the efficiency limiting factors. A systematic methodology is developed and integrated into the proposed model to simulate the characteristics in the quantum efficiency. The proposed model is demonstrated with respect to an ideal device model under a set of bias conditions to selectively deactivate performance limiting parameters under light and voltage biased conditions. Under particular wavelength regions and bias conditions, a particular type of defects near the heterojunction interface significantly impact the carrier collection of devices. This deep acceptor type defect distribution is located in the band of $+/-0.3 \mathrm{eV}$ from the midgap. These defect states influence CZTSSe spectral responses of red and IR light wavelength regions in quantum efficiency caused by affected depletion width toward the back contact. Therefore, the quantum efficiency of CZTSSe devices is altered disproportionally at biased conditions.
\end{abstract}

Key words: Degradation, thin film solar cells, defects, modeling.

\section{Introduction}

Q-E (quantum efficiency) measurements are a popular and practical method to understand the photocarrier generation and loss mechanism in optoelectronic devices such as detectors, thin film solar cell. Quantum efficiency is defined as the ratio of the number of extracted charge carriers to the number of incident photons. IQE (internal quantum efficiency) is the ratio of the number of charge carriers extracted from the cell to the number of photons absorbed in the active layer whereas external quantum efficiency includes reflection loss. To model quantum efficiency, the analytical model is accounted for the simplified set of parameters to fit while the numerical simulation requires a number of parameters to extract the quantum

Corresponding author: Sanghyun Lee, Ph.D., professor, research fields: device physics, photovoltaics and flash memory. efficiency model. With these critical parameter sets, modeling Q-E deepens the understanding of the recombination process as the magnitude of $\mathrm{Q}-\mathrm{E}$ is inversely proportional to the recombination process. Moreover, the spectral response of Q-E provides information about the spatial distribution of the charge recombination path. Although P-type CZTSSe (copper zinc tin sulphur selenium) thin film solar cells have proven a number of advantages over conventional solar cells such as high absorption coefficient $\left(>10^{4} \mathrm{~cm}^{-1}\right)$ and ideal direct bandgap $(1.4 \sim 1.5 \mathrm{eV})$ with earth abundant low cost materials which enable a perfect platform for high-efficient low-cost photovoltaic application [1-5], CZTSSe solar cells suffer from severe recombination loss showing less than $12.6 \%$ efficiency $[1,6]$. CZTSSe is also called kesterite solar cells following the crystal structure including CZTS (copper zinc tin sulphur) and CZTSe (copper zinc tin 
selenium) solar cells devices. Conversely, similar thin film solar cells show a dramatic improvement over the last five years. The best efficiency from zincblende (CdTe) solar cells is $21.5 \%$ and chalcopyrite (CIGS) solar cells is $21.7 \%$ ZSW. However, the maximum theoretical efficiency of CZTSSe solar cells based on the Shockley-Queisser limit is about 32.2\% which implies there is much room for the improvement [7]. Since the QE spectral responses can provide the information to identify the loss mechanism of CZTSSe solar cells, Q-E modeling and measurements are highly required to achieve the recombination path information and passivation to maximum possible efficiency.

There are different types of recombination paths to lower the carrier collection process at different interfaces such as buffer/window, heterojunction, and absorber/back contact. At these interfaces, recombination active defects are present due to intrinsic symmetry effects [8], lattice mismatch, and segregation of impurities aggravated by grain boundaries, which are inevitable for polycrystalline solar cells. The recombination current enhances forward diode current which deteriorates the conversion efficiency.

One of the device parameters which attribute the deficiency in conversion efficiency is the open circuit voltage (Voc) that accounts for the forward bias on the solar cell due to the bias of the solar cell junction with the light-generated current. This is a point where diode current is equivalent to light-generated current, affected by the saturation current of the solar cell. An important effect comes from the saturation current, which depends on recombination in the solar cell and can vary by orders of magnitude. Therefore, Voc depends on the recombination in the device. Diode saturation current is a function of bandgap increasing with bandgap. Despite a recent significant improvement, one of the most fundamental problems that remain is lower Voc $[9,10]$. Moving Voc beyond $750 \mathrm{mV}$ in CZTSSe solar cells requires improvements in both recombination and built-in potential of the junction. Firstly, the recombination currents provide the dominant contribution to the saturation current and hence, the reduction of the recombination current can result in higher Voc. Secondly, the built-in potential of the junction is determined by the doping levels in the heterojunction participating layers. The higher the doping concentration is, the higher the Voc. In addition, the work function of contact metals and the back contact barrier affect Voc which is well published elsewhere $[11,12]$.

\section{Experminetal Method}

The spectral response of CZTSSe thin film solar cells is studied with quantum efficiency device modeling at particular wavelengths to understand the efficiency limiting factors. This will also provide the proper interpretation of device behaviors when a device is measured by quantum efficiency at different temperatures, for instance, $200 \mathrm{~K}$ and $300 \mathrm{~K}$. We simulated quantum efficiency by utilizing SCAPS simulator version 3.2.01. In this work, we focus on the efficiency limiting factor by investigating the influence of near-interface defect states on quantum efficiency at particular bias conditions with the intention of evaluating peculiar quantum efficiency responses by defect distributions with adjusting numerical parameters. Furthermore, we simulated the temperature-dependent spectral response of quantum efficiency for the same defect model.

Input parameters in each material are thickness, relative permittivity, electron mobility, hole mobility, acceptor concentration, donor concentration, band gap, and effective density of states. The modeled device structure of CZTSSe thin film solar cell is with $\mathrm{ZnO}$ window layer, CdS buffer, CZTSSe absorber, and Molybdenum (Mo) back contact. In Table 1, input parameters are illustrated for the reference. To avoid the complexity of interface defects at absorber/back contact, absorber/buffer, and buffer/window, we adopted the basic input parameters from literature, theories, or reasonable estimates [13-16]. 
Table 1 Parameter set used in this work and types of defects (a: acceptor, d: donor, n: neutral).

\begin{tabular}{lllll}
\hline Parameters & Symbol (unit) & ZnO & CdS & CZTSSe \\
\hline Thickness & $\mathrm{d}(\mathrm{nm})$ & 200 & 50 & 2,500 \\
Band gap & $\mathrm{E} g(\mathrm{eV})$ & 3.3 & 2.4 & $1.2-1.3$ \\
Electron affinity & $\chi(\mathrm{eV})$ & 4.4 & 4.2 & 4.1 \\
Relative permittivity & $\epsilon / \epsilon_{\mathrm{r}}$ & 9 & 10 & 10 \\
Effective density of state & $\mathrm{N}_{\mathrm{C}}\left(10^{18} \mathrm{~cm}^{-3}\right)$ & 2.2 & 2.2 & 2.2 \\
Effective density of state & $\mathrm{N}_{\mathrm{V}}\left(10^{19} \mathrm{~cm}^{-3}\right)$ & 1.8 & 1.8 & 1.8 \\
Electron thermal velocity & $v_{n}\left(10^{7} \mathrm{~cm} / \mathrm{s}\right)$ & 1.0 & 1.0 & 1.0 \\
Hole thermal velocity & $v_{p}\left(10^{7} \mathrm{~cm} / \mathrm{s}\right)$ & 1.0 & 1.0 & 1.0 \\
Electron mobility & $\mu_{n}\left(\mathrm{~cm}^{2} / \mathrm{V}\right)$ & 100 & 100 & 25 \\
Hole mobility & $\mu_{p}\left(\mathrm{~cm}^{2} / \mathrm{V}\right)$ & 25 & 25 & 0 \\
Donor concentration & $\mathrm{N}_{\mathrm{D}}\left(10^{17} \mathrm{~cm}^{-3}\right)$ & 10.0 & 1.0 & 2.0 \\
Acceptor concentration & $\mathrm{N}_{\mathrm{A}}\left(10^{14} \mathrm{~cm}^{-3}\right)$ & 0 & 0 & $\mathrm{~d} / \mathrm{a} / \mathrm{n}$ \\
Defect type & $\mathrm{a}, \mathrm{d}, \mathrm{n}$ & $\mathrm{d}$ & $\mathrm{a}$ & \\
\hline
\end{tabular}

\section{Results and Discussion}

\subsection{Ideal Trap Free Case}

For the baseline study, the initial simulations of both $\mathrm{I}-\mathrm{V}$ (current-voltage) and Q-E (quantum efficiency) have been done without traps near the heterojunction interface. The default illumination spectrum condition is set to standard AM 1.5 and operation temperature are set to $300 \mathrm{~K}$. A representative result of the simulated $\mathrm{I}-\mathrm{V}$ curve of a CZTSSe device without heterojunction defects is demonstrated in Fig. 1a. As anticipated, an ideal steep I-V curve leads to conversion efficiency, $15.3 \%$ with the emphasis on higher Voc $(661 \mathrm{mV})$ and FF (72.5\%) than any reported emprical Voc and FF due to the intrinsic defect-free interface. The trend of an ideal device in Fig. 1a is explained elsewhere [17] that hetero-interface collection efficiency with voltage is due only to bias-dependent interface recombination losses occurring in regions that are thin compared to the absorption length $1 / \alpha$. Therefore, short-circuit conductance at zero-bias can be used to evaluate interface recombination losses at any combination of electrical bias and wavelength as shown below.

$$
\begin{gathered}
J=J_{0}\left\{\exp \left(\frac{q V}{n k T}\right)-1\right\}-H(\lambda, V) \\
\left.\frac{d J}{d V}\right|_{V=0}=\left.\left.\frac{1}{R}\right|_{V=0} \approx J_{L 0} \frac{d h}{d V}\right|_{V=0}
\end{gathered}
$$

where, $J$ is current density, $J_{0}$ is reverse saturation current, $n=$ ideality factor, $k$ is boltzman constant, $T$ is temperature, $\lambda$ is a wavelength, $V$ is voltage, $J_{L 0}$ is the $H=1$ value of the light current reverse saturation current, and $H(\lambda, V)$ is a collection function written as the product, $g(\lambda, V) \cdot h(V)$, which are the bulk recombination/absorption and the bias dependence of interfacial recombination, respectively [17]. With the presence of a reasonable depletion width that can accommodate the generation of charge carriers, the short circuit conductance is affected by the interfacial recombination term, $h(V)$. Accordingly, the simulated I-V curve in Fig. 1b shows significant Voc improvement by showing nearly zero slope of $\mathrm{I}-\mathrm{V}$ curve at short circuit condition $\left(<2.6 \mathrm{mS} / \mathrm{cm}^{2}\right)$ which can be justified with the alleviated recombination. The total forward diode current can be decreased by lowered recombination current by showing the ideal slope at the short circuit current condition [17]. Consequently, the resulting quantum efficiency is demonstrated in Fig. 1c, which demonstrates the simulated quantum efficiency of the same device with the highest efficiency as a function of applied bias voltage. At zero bias, good carrier collection is observed at most of the wavelength regions. However, the carrier collection deteriorates as applied bias increases, caused by reduced depletion width of absorber by applied biases. To confirm the uniformity of reduced carrier collection at each wavelength region, we compare carrier collection 


\section{Mo back contact \\ CZTSSe (2.5um) \\ CdS (50nm) \\ $\mathrm{ZnO}(200 \mathrm{~nm})$ \\ Front contact}

(a)

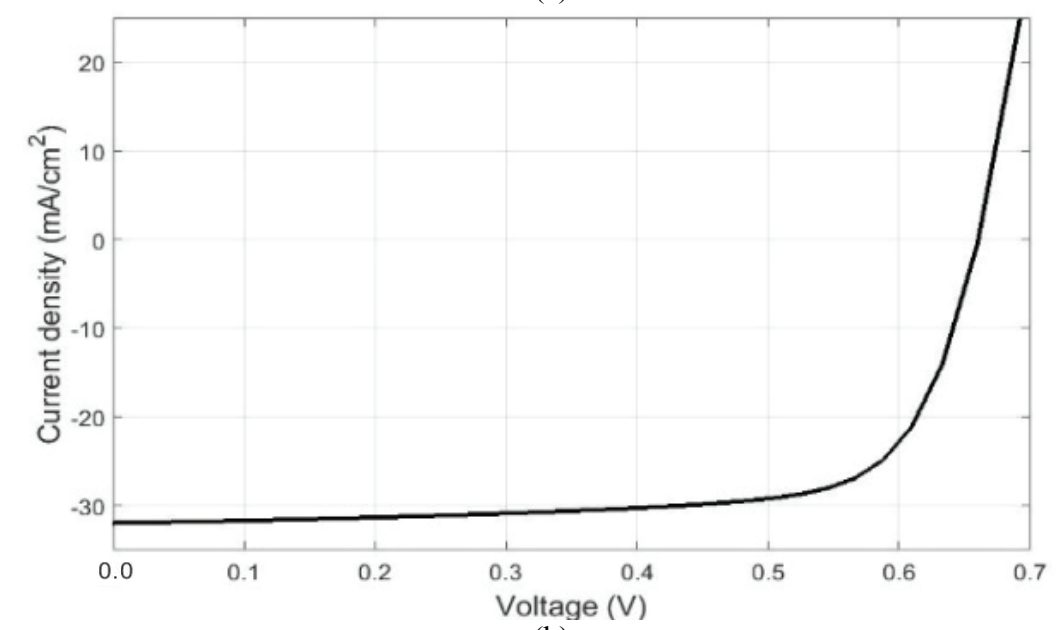

(b)

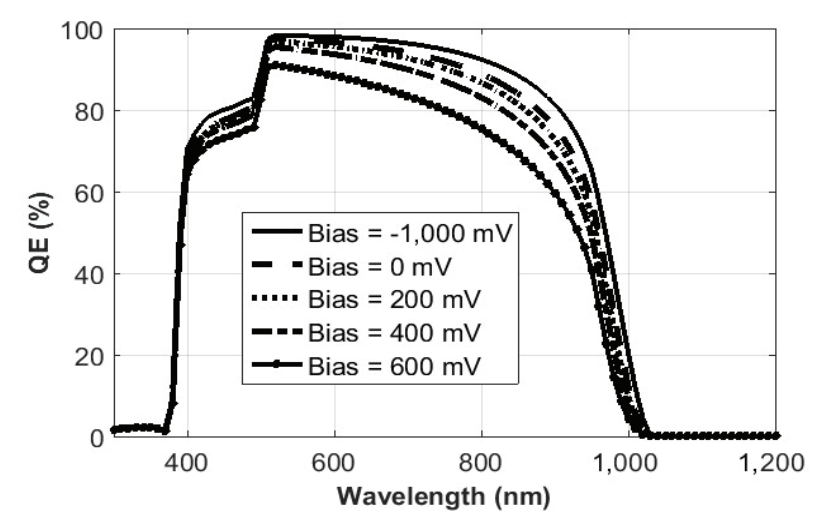

(c)

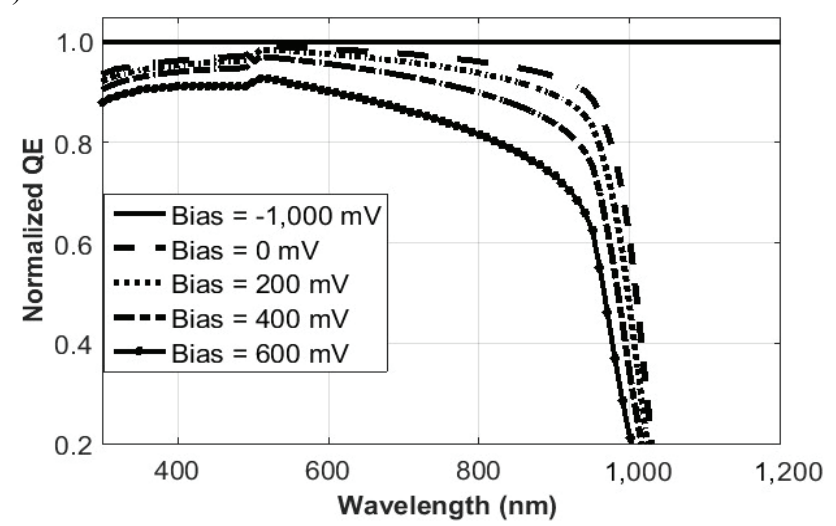

(d)

Fig. 1 I-V and Q-E of ideal CZTSSe solar cell without interface or near-interface defects, (a) structure of CZTSSe solar cell used in this simulation, (b) I-V with efficiency $(15.3 \%)$, Voc $(661 \mathrm{mV})$, FF $(72.5 \%)$, Jsc $\left(32.0 \mathrm{~mA} / \mathrm{cm}^{2}\right)$, (c) Q-E at biased conditions $(-1.0 \mathrm{~V}, 0.0 \mathrm{~V}, 0.2 \mathrm{~V}, 0.4 \mathrm{~V}, 0.6 \mathrm{~V})$, (d) normalized Q-E (Q-E at each bias divided by Q-E at full collection, $-1 \mathrm{~V})$.

at each bias with full collection by applying negative bias, $-1 \mathrm{~V}$ [17]. In Fig. 1d, each simulated Q-E at each bias $(0,200,400$, and $600 \mathrm{mV})$ is normalized by full carrier collection with Q-E at $-1 \mathrm{~V}$. From the normalized Q-E, we concluded that the shape of the normalized Q-E response is similar to the spectral response of the device without normalization which has ideal uniform doping and defect throughout the absorber layer. In other words, lateral effects in the absorber, for instance, horizontal doping fluctuation in Fig. 1a, is ruled out to avoid the complexity of modeling. Furthermore, the bias dependent normalized Q-E response shows the nearly uniform collapse of quantum efficiency by keeping their curve shape over the wide range of wavelengths with the trend of increasing absorber collection loss due to reduced 
depletion width by applied biases near $(500-800 \mathrm{~nm})$ and $(800-1,033 \mathrm{~nm})$.

\subsection{Trends by Various Interface Defect Distributions}

The simulation with near-interface defects begins with placing acceptor and donor type defects near both conduction and valence bands as well as midgap. The SCAPS simulator allows three different parameter sets of interface defects for both acceptor and donor types that significantly affect the device performance (capture cross sections, defect energy level Et, defect density). However, realistic CZTSSe devices consist of complex multilayers with defects and/or defect compounds at the interface between CZTSSe and CdS. Moreover, it is widely accepted the direct measurement of this layers' electronic properties is difficult due to complicated window structures with $\mathrm{CdS}, \mathrm{ZnO}$, and TCO layers inter-diffusion. Hence, we focus on main effects in quantum efficiency when a thin defective interface layer between CdS and CZTSSe is added in this study. For consistency with reported literature, theories, or reasonable estimates, capture cross sections are fixed at $1.0 \times 10^{-12} \mathrm{~cm}^{2}$ for electrons and $1.0 \times 10^{-15}$ $\mathrm{cm}^{2}$ for holes at donor type defect and at $1.0 \times 10^{-15} \mathrm{~cm}^{2}$ for electrons and $1.0 \times 10^{-12} \mathrm{~cm}^{2}$ for holes at acceptor type defects [12-15].

For this simulation study, we insert the thin $5 \mathrm{~nm}$ defective interface layer between CdS and CZTSSe as a representation of the interface or near-interface defects. Input parameters are set to the same as those of CdS except thickness and doping densities which are set to the same values of $1 \times 10^{14} \mathrm{~cm}^{-3}$ for donor and acceptor assuming the intermixing of n-type dopant (CdS) and p-type dopant (CZTSSe).

\subsection{Impact of Defect Concentration and Energy States}

In this section, we intend to clarify peculiarities that we observed in our modeling and simulations as well as reported data by surveying device parameters. Before surveying the bias-impact on devices, we begin with investigating the trend across a set of defect concentrations in or near the heterojunction interface, represented by an additional defective interface layer. Fig. 2 shows the simulated quantum efficiency with an inserted defective interface layer between CZTSSe and $\mathrm{CdS}$ buffer layers as a function of defect concentration. The simulation begins with defect density, $1.0 \times 10^{15}$ $\mathrm{cm}^{-3}$ in this layer as a deep acceptor type bulk defect. We located this type of defect between $0.9 \mathrm{eV}$ and 1.5 $\mathrm{eV}$ above the top of the valence band around the midgap $(1.2 \mathrm{eV})$. As defect concentration increases to $1.0 \times 10^{17} \mathrm{~cm}^{-3}$, we could not find particularly stronger carrier collection losses in any specific wavelength regions. However, we observed serious collection loss at the absorber is demonstrated in region 2 (500-800 $\mathrm{nm})$ and $3(800-1,033 \mathrm{~nm})$ once we increase defect concentration to $>6.0 \times 10^{17} \mathrm{~cm}^{-3}$. Slight collection loss is found at 300-500 nm with slight inflection of collection near $500 \mathrm{~nm}$. This simulated peculiar spectral response is described in detail in the following secions.

\subsection{Temperature Dependence}

To model this peculiarity, there are two possible scenarios for the weaker reduction in spectral response near 500-550 nm which is near-interface region and stronger collapse at $>500 \mathrm{~nm}$ region as the bias

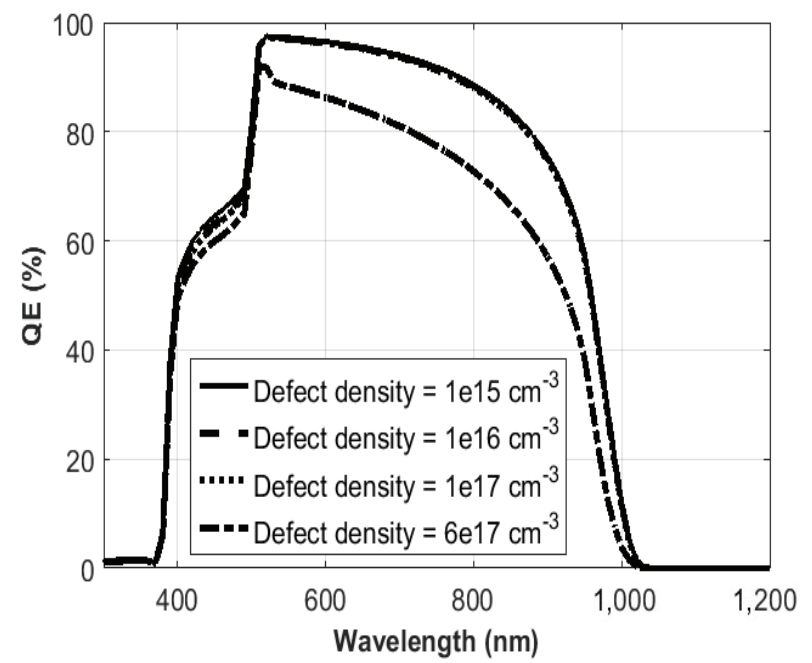

Fig. 2 Q-E of CZTSSe device with the inserted interface defective layer between CdS and CZTSSe, varying acceptor type defect density from $1.0 \times 10^{15} \mathrm{~cm}^{-3}$ to $6.0 \times 10^{17} \mathrm{~cm}^{-3}$. 


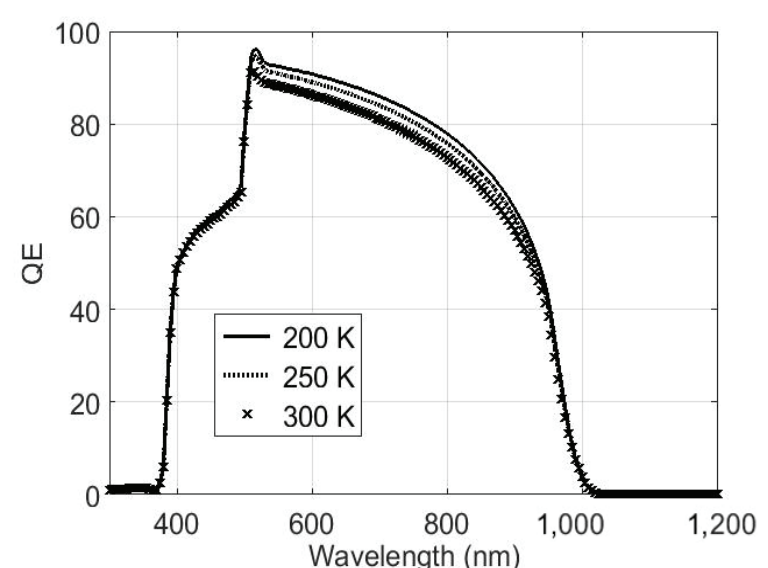

Fig. 3 Quantum efficiency of CZTSSe solar cell with defective interface layer $\left(6.0 \times 10^{17} \mathrm{~cm}^{-3}\right)$ at $200 \mathrm{~K}, 250 \mathrm{~K}$, and $300 \mathrm{~K}$. The intensified spectral response between $500-550 \mathrm{~nm}$ at $250 \mathrm{~K}$ and $200 \mathrm{~K}$ remains the same as $300 \mathrm{~K}$.

increases from $-1 \mathrm{~V}$ (full collection) to $0 \mathrm{~V}$ (short circuit condition). The response $(<500 \mathrm{~nm})$ from the front contact area (ex. buffer and TCO) is ignored due to the modeling complexity as well as lesser sensitivity due to higher doping concentration (TCO). In the first scenario, the quasi-neutral region of the absorber is far more sensitive to the electric field reduction from the full collection electric field due to near-interface acceptor type defects with the modified weaker electric field. For the second scenario, which is a most probable scenario, the electric field at the heterojunction interface is greater than the electric field at the absorber and reduced less at the applied greater bias by the inserted defect state distributions. To check the difference in scenarios, we now compare three different temperatures to reduce the ionization probability of all the charge carrier states to confirm any stronger energy states that tend to have the higher possibility of ionization by different temperature. As temperature decreases from $300 \mathrm{~K}$ to $200 \mathrm{~K}$, the spectral response from the same device with defect concentration, $6.0 \times 10^{17} \mathrm{~cm}^{-3}$ with energy states between $0.9 \mathrm{eV}$ and $1.5 \mathrm{eV}$ is illustrated in Fig. 3. As expected, the reduction in temperature reduces the net dominant recombination mechanism which also improves Voc [18]. Consequently, the spectral responses in Q-E increases as temperature decreases from $300 \mathrm{~K}$ to $200 \mathrm{~K}$. The regions from $500 \mathrm{~nm}$ to 900 $\mathrm{nm}$ show the Q-E reduction compared to a slight change in the other regions. However, the intensified spectral response between $500-550 \mathrm{~nm}$ remains the same at lower temperatures as $300 \mathrm{~K}$ compared to the rest of wavelength regions at the same compared temperature. In other words, the shape of curves is kept the same at lower temperatures, indicating the overall recombination mechanism of the absorber quasi-neutral and the interface is decreased by lower temperatures. Consequently, the quantum efficiency increases as temperature goes down.

\subsection{The Impact of Light and Voltage Biases}

The described tendencies of defect concentrations depend also on the defect energy state as well as defect concentrations. With only increasing the energy state of acceptor type defect distributions from the valence band or decreasing from the conduction band to $0.9 \mathrm{eV}$ $\sim 1.5 \mathrm{eV}$, this peculiar spectral response with the inflection near $>500 \mathrm{~nm}$ becomes stronger. On the other hand, increasing the energy state of donor type defect distributions in those energy states shows a weak dependence on this peculiarity. Hence, we set defect concentration at $6.0 \times 10^{17} \mathrm{~cm}^{-3}$ as deep acceptor type defects $+/-0.3 \mathrm{eV}$ from the midgap and the resulting biased quantum efficiency is shown in Fig. 4. As expected, the quantum efficiency at $-1 \mathrm{~V}$ (not shown), over all wavelength regions indicates similar spectral response compared to that of the device without defects in Fig. 1c as $-1 \mathrm{~V}$ bias provides the possible full collection at heterojunction [17]. As applied bias reaches until $0 \mathrm{~V}$, slight absorber loss is observed similar to Fig. 1c. However, once bias increases above $0.2 \mathrm{~V}$, the stronger collapse of Q-E response occurs at both $<500 \mathrm{~nm}$ and $>550 \mathrm{~nm}$ as compared to the region 500-550 $\mathrm{nm}$. However, the reduction of quantum efficiency between $500-550 \mathrm{~nm}$ is milder than the other regions. This peculiar Q-E response occurs with a spike near $520-530 \mathrm{~nm}$ in the blue light region which is also reported in their measurements in literature $[19,20]$. 


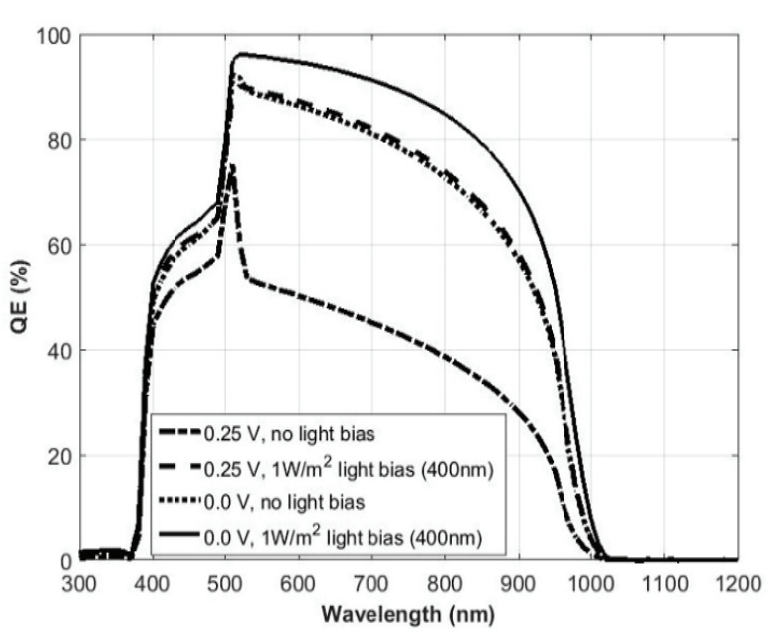

Fig. 4 Quantum efficiency of CZTSSe solar cell with defective interface layer at $0 \mathrm{~V}$ and $0.25 \mathrm{~V}$ with and without light biased (0.01 sun, $1 \mathrm{~W} / \mathrm{m}^{2}$, at $\left.400 \mathrm{~nm}\right)$ at $0 \mathrm{~V}$ and $0.25 \mathrm{~V}$.

With inserted deep acceptor type defects near the heterojunction interface layer, we are able to explain the aggravated quantum efficiency drop in $<500 \mathrm{~nm}$ and $>550 \mathrm{~nm}$. Considering the presence of higher defect concentration in this heterojunction interface, the unionized acceptor type defect distributions between $0.9 \sim 1.5 \mathrm{eV}$ around the midgap influence the electric field and hence space charge region of absorber toward the back contact. Accordingly, higher electric field near the heterojunction interface facilitates the increased absorption by a front part of the absorber near CdS layer. However, this higher electric field decreases absorption by the majority of CZTSSe absorber toward the back contact. Hence, increasing voltage bias to $0.2 \mathrm{~V}$ and $0.2 \mathrm{~V}$ further reduces the depletion width even after shortened depletion width with higher interface defects. To eliminate interface defect effects, applying weak blue light bias (400nm) can compensates ionized defects by trapping holes from the valence band by verifying the spectral response at $>550 \mathrm{~nm}$ in quantum efficiency. Using this model, weak light bias $\left(1 \mathrm{~W} / \mathrm{m}^{2}, 0.01 \mathrm{sun}\right)$ is applied to the same device at $0 \mathrm{~V}$ and $0.25 \mathrm{~V}$ in Fig. 4 and highlighted with normalized Q-E for the comparison between $0.25 \mathrm{~V}$ and $0 \mathrm{~V}$ in Fig. 5. Weak blue light bias onto window and buffer layers almost fully recovers CZTSSe absorber carrier collection even at $0.25 \mathrm{~V}$.
Excluding noises in the regions of $<400 \mathrm{~nm}$ and $>$ $1,000 \mathrm{~nm}$, normalized Q-E in Fig. 4 clearly indicates weak blue light bias recovers the carrier collection of absorber near $>550 \mathrm{~nm}$ at both $0 \mathrm{~V}$ and $0.25 \mathrm{~V}$ with small increase near $<500 \mathrm{~nm}$. At $0.25 \mathrm{~V}$, the depletion width with light bias is larger than that of without weak blue light bias. Therefore, we can confirm the compensation of ionized deep near-interface defects between $0.9 \mathrm{eV}$ and $1.5 \mathrm{eV}$ and recovers the depletion width which increases carrier collection in CZTSSe absorber.

The presence of deep acceptor type bulk defects $(0.9$ $\mathrm{eV} \sim 1.5 \mathrm{eV})$ in the defective interface layer can effectively decreases the depletion width near the heterojunction interface by increasing negative charge density, which only can be compensated by additional bias light. Without light bias in Fig. 6a, the defective interface layer impacts the electric field of the quasi-neutral region of the absorber layer by pinning the electric field of the interface layer at its maximum within the device induced due to inserted near-interface defect states. The quantum efficiency at the interface is subject to be intensified due to this pinned interface layer. Once the light bias is applied to this defective interface layer, interface defect states will be compensated with generated holes which increase the

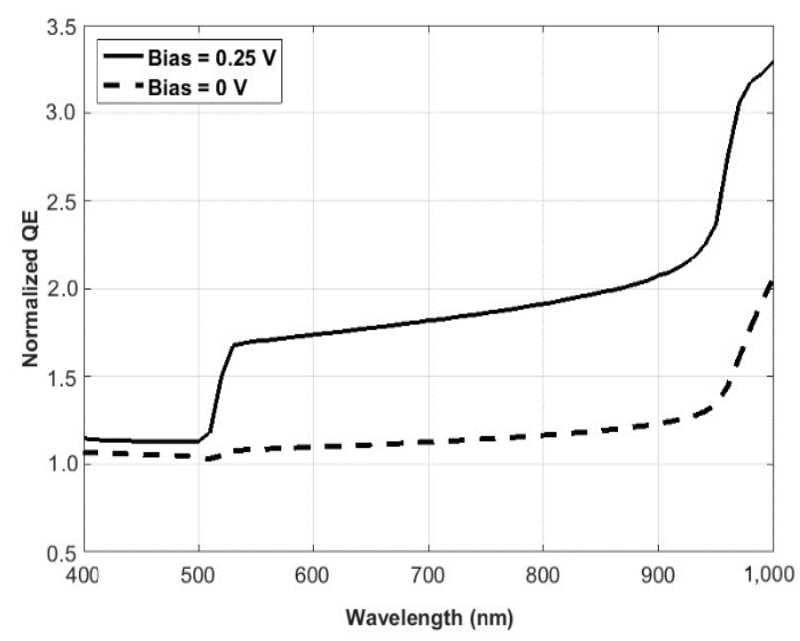

Fig. 5 Normalized Q-E (Q-E at blue light bias divided by $\mathrm{Q}-\mathrm{E}$ at no light bias) with defective interface layer at $0 \mathrm{~V}$ and $0.25 \mathrm{~V}$ when weak blue light bias $\left(0.01 \mathrm{sun}, 1 \mathrm{~W} / \mathrm{m}^{2}\right.$ at 400 $\mathrm{nm}$ ) is present. 


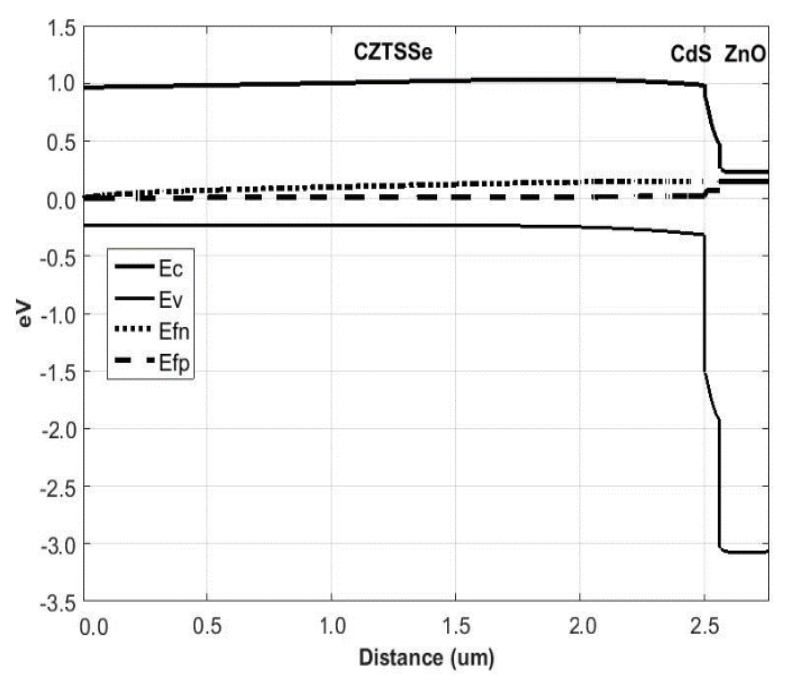

(a)

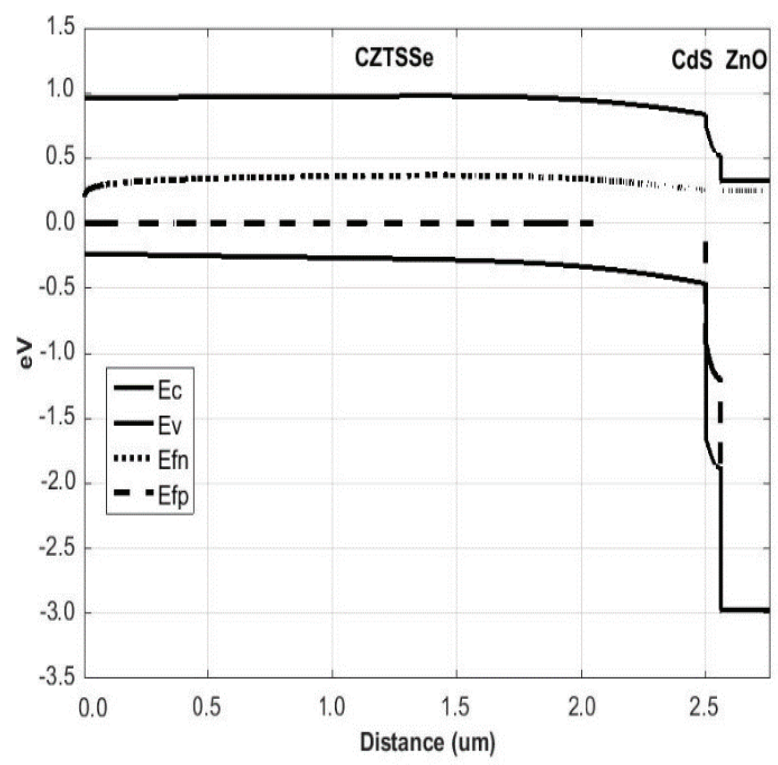

(b)

Fig. 6 Light biased $\left(1 \mathrm{~W} / \mathrm{m}^{2}, 0.01\right.$ sun at $\left.400 \mathrm{~nm}\right)$ Q-E of CZTSSe solar cell with defective interface layer at $0 \mathrm{~V}$ and $0.25 \mathrm{~V}$, (a) band diagram without light bias at $0.25 \mathrm{~V}$, (b) band diagram with light bias at $0.25 \mathrm{~V}$, showing wider depletion width and higher quasi Fermi level $\left(E_{F n}\right)$ than (a) at light bias at $0.25 \mathrm{~V}$.

depletion width toward the back contact in Fig. 6 b. Hence, the depletion width of the absorber is wider than the device in Fig. 6a. The band diagram and carrier density of holes and electrons indicate higher electric field with less hole concentration at the interface, resulting in smaller depletion width in the presence of the defective interface layer.

\section{Conclusions}

We have systematically modeled quantum efficiency responses of CZTSSe thin film solar cell devices at different temperatures and under a set of biased conditions by comparing a defect-free ideal device and a device with defective interface layer. For CZTSSe solar cells, it is assumed that deep acceptor type near-heterojunction interface defects from $0.9 \mathrm{eV}$ $\sim 1.5 \mathrm{eV}$ above the top of the valence band are difficult to be compensated which provide additional negative charges in space charge regions. These additional ionized defects provide additional doping to the heterojunction interface which cramp the depletion width and hence, weaker spectral responses near red and IR regions. This trend is intensified with biased Q-E measurements, which will guide the direction of future research in CZTSSe solar cells device modeling and experiments. Although it is clear that repetitive measurements need to be carried out to explain simulation results on the same cells, this study shows fairly good agreement with numerical calculation and reported experimental results.

\section{Acknowledgement}

This project is partially supported by the National Science Foundation under Cooperative Agreement No. 1355438.

\section{References}

[1] Wang, W., Winkler, M. T., Gunawan, O., Gokmen, T., Todorov, T. K., Zhu, Y., and Mitzi, D. B. 2014. "Device Characteristics of CZTSSe Thin-Film Solar Cells with 12.6\% Efficiency." Advanced Energy Materials 4 (7): 1301465 .

[2] Mitzi, D. B., Todorov, T. K., Wang, K., and Gunha, S. 2011. "The Path towards a High-Performance Solution-Processed Kesterite Solar Cell." Solar Energy Materials and Solar Cells 95 (6): 1421-36.

[3] Kumar, Y. K., Babu, G. S., Bhaskar, P. U., and Raja, V. S. 2009. "Preparation and Characterization of Spray-Deposited $\mathrm{Cu}_{2} \mathrm{ZnSnS}_{4}$ Thin Films." Solar Energy Materials and Solar Cells 93 (8): 1230-7.

[4] Chan, C., Lam, H., and Surya, C. 2010. "Preparation of $\mathrm{Cu}_{2} \mathrm{ZnSnS}_{4}$ Films by Electrodeposition Using Ionic 


\section{Bias Dependence by SCAPS Simulation}

Liquids." Solar Energy Materials and Solar Cells 94 (2): 207-11.

[5] Katagiri, H., Jimbo, K., Yamada, S., Kamimura, T., Maw, W., Fukano, T., Ito, T., and Motohiro, T. 2008. "Structural and Optical Properties of the New Absorber $\mathrm{Cu}_{2} \mathrm{ZnSnS}_{4}$ Thin Films Grown by Vacuum Evaporation Method." Journal of Applied Physics 1: 41201-2.

[6] Jackson, P., Hariskos, D., Wuerz, R., Wischmann, W., and Powalla, M. 2014. "Compositional Investigation of Potassium Doped $\mathrm{Cu}(\mathrm{In}, \mathrm{Ga}) \mathrm{Se}_{2}$ Solar Cells with Efficiencies Up to 20.8\%." Physica Status Solidi (RRL)-Rapid Research Letters 8 (3): 219-22.

[7] Shockley, W., and Queisser, H. K. 1961. "Detailed Balance Limit of Efficiency of p-n Junction Solar Cells." Journal of Applied Physics 32: 510-9.

[8] Lucovsky, G., Lee, S., Long, J. P., Seo, H., and Lüning, J. 2009. "Interfacial Transition Regions at Germanium/Hf Oxide Based Dielectric Interfaces: Qualitative Differences between Non-Crystalline $\mathrm{Hf} \mathrm{Si}$ Oxynitride and Nanocrystalline $\mathrm{HfO}_{2}$ Gate Stacks." Microelectronic Engineering 86: 224-34.

[9] Mitzi, D. B., Gunawan, O., Todorov, T. K., and Barkhouse, D. A. R. 2013. "Preparation of $\mathrm{Cu}_{2} \mathrm{ZnSnS}_{4}$ Films by Electrodeposition Using Ionic Liquids." Philosophical Transactions of the Royal Society A: Mathematical, Physical and Engineering Sciences 371: 20110432, 1-22.

[10] Gunawan, O., Todorov, T. K., and Mitzi, D. B. 2010. "Loss Mechanisms in Hydrazine-Processed $\mathrm{Cu}_{2} \mathrm{ZnSn}(\mathrm{Se}, \mathrm{S})_{4}$ Solar Cells." Applied Physics Letters 97 (23): 233506, 1-3.

[11] Jager, T., Romanyuk, Y. E., Bissig, B., Pianezzi, F., Nishiwaki, S., Reinhard, P., Steinhauser, J., Schwenk, J., and Tiwari, A. N. 2015. "Improved Open-Circuit Voltage in $\mathrm{Cu}(\mathrm{In}, \mathrm{Ga}) \mathrm{Se}_{2}$ Solar Cells with High Work Function Transparent Electrodes." Journal of Applied Physics 117 (22): 225303, 1-5.

[12] Haug, F.-J., Biron, R., Kratzer, G., Leresche, F., Besuchet, J., Ballif, C., Dissel, M., Kretschmer, S., Soppe, W.,
Lippens, P., and Leitner, K. 2012. "Improvement of the Open Circuit Voltage by Modifying the Transparent Indium Tin Oxide Front Electrode in Amorphous Nip Solar Cells." Progress in Photovoltaics 20 (6): 727-34.

[13] Simya, O. K., Mahaboobbatcha, A., and Balachander, K. 2015. "A Comparative Study on the Performance of Kesterite Based Thin Film Solar Cells Using SCAPS Simulation Program." Superlattices and Microstructures 82 (June): 248-61.

[14] Zhao, W., Zhou, W., and Miao, X. 2012. "Numerical Simulation of CZTS Thin Film Solar Cell." In Proceedings of the 7th IEEE International Conference on Nano/Micro Engineered and Molecular Systems (NEMS), 502-5.

[15] Gokmen, T., Gunawan, O., and Mitzi, D. B. 2000. "Modelling Polycrystalline Semiconductor Solar Cells." Thin Solid Films 361 (February): 527-32.

[16] Polizzotti, A., Repins, I. L., Noufi, R., Wei, S.-H., and Mitzi, D. B. 2013. "The State and Future Prospects of Kesterite Photovoltaics." Energy and Environmental Science 6 (11): 3171-82.

[17] Fahrenbruch, A. L., and Bube, R. H. 1983. Fundamentals of Solar Cells: Photovoltaic Solar Energy Conversion. St Louis: Academic Press, 231-4.

[18] Thompson, C., Hegedus, S., Shafarman, W., and Desai, D. 2008. "Temperature Dependence of $\mathrm{V}_{\mathrm{OC}}$ in CdTe and $\mathrm{Cu}(\mathrm{InGa})(\mathrm{SeS})_{2}$-Based Solar Cells." In Proceedings of the 33rd IEEE Photovoltaic Specialists Conference, 1-6.

[19] Gokmen, T., Gunawan, O., and Mitzi, D. B. 2014. "Semi-Empirical Device Model for $\mathrm{Cu}_{2} \mathrm{ZnSn}(\mathrm{S}, \mathrm{Se})_{4}$." Applied Physics Letters 105 (3): 033903, 1-5.

[20] Brammertz, G., Oueslati, S., Buffiere, M., Bekaert, J., Anzeery, H. E., Messaoud, K. B., Sahayarj, S., Nuytten, T., Koble, C., Meuris, M., and Poortmans, J. 2015. "Investigation of Properties Limiting Efficiency in $\mathrm{Cu}_{2} \mathrm{ZnSn}(\mathrm{S}, \mathrm{Se})_{4}$-Based Solar Cells." IEEE Journal of Photovoltaics 5 (2): 649-55. 